\title{
Correction to: Dodging bullets: The heterogeneous effect of political violence on greenfield FDI
}

\author{
Caroline T Witte ${ }^{1,2}$, \\ Martijn J Burger ${ }^{2}$, \\ Elena I lanchovichina ${ }^{3}$ and \\ Enrico Pennings ${ }^{2}$
}

\footnotetext{
${ }^{1}$ Department of Strategic Management and Globalization, Copenhagen Business School, Kilevej 14, 2000 Frederiksberg, Denmark; ${ }^{2}$ Department of Applied Economics, Tinbergen Institute and Erasmus Research Institute of Management (ERIM), Erasmus University, Rotterdam, PO Box 1738, 3000 DR Rotterdam, The Netherlands; ${ }^{3}$ Chief Economist Office, Middle East and North Africa Region, The World Bank, Washington, DC, USA
}

Correspondence:

CT Witte, Department of Strategic Management and Globalization, Copenhagen Business School, Kilevej 14, 2000 Frederiksberg, Denmark e-mail: witte@ese.eur.nl
Journal of International Business Studies (2019) 50, 454-455. https://doi.org/| 0.1057/s41267-018-0200-6

\section{CORRECTION TO: JOURNAL OF INTERNATIONAL BUSINESS STUDIES (2017) 48, 862-892 https://doi.org/10.1057/s41267-017-0079-7}

Owing to a production error, the coefficients of the BRD (ln) * localized and Greenfield FDI variables were not correctly displayed in the even columns of Table 2 in the original article. A corrected version of this table is provided here. The corrected values are italicized. 
Table 2 Effect of political conflict on FDI by relatively undiversified and diversified firms

\begin{tabular}{|c|c|c|c|c|c|c|}
\hline & \multicolumn{6}{|c|}{ Dependent variable: log greenfield FDI (in USD millions), LSDV estimation } \\
\hline & \multicolumn{2}{|c|}{ Total FDI } & \multicolumn{2}{|c|}{ Resource-related FDI } & \multicolumn{2}{|c|}{ Non-resource-related FDI } \\
\hline & $\begin{array}{l}\text { Undiversified } \\
\text { MNEs } \\
\text { (1) }\end{array}$ & $\begin{array}{l}\text { Diversified } \\
\text { MNEs } \\
(2)\end{array}$ & $\begin{array}{l}\text { Undiversified } \\
\text { MNEs } \\
\text { (3) }\end{array}$ & $\begin{array}{l}\text { Diversified } \\
\text { MNEs } \\
(4)\end{array}$ & $\begin{array}{l}\text { Undiversified } \\
\text { MNEs } \\
(5)\end{array}$ & $\begin{array}{l}\text { Diversified } \\
\text { MNEs } \\
(6)\end{array}$ \\
\hline $\mathrm{BRD}_{t-1}(\ln )$ & $\begin{array}{l}-0.129^{+} \\
(0.073)\end{array}$ & $\begin{array}{l}-0.015 \\
(0.082)\end{array}$ & $\begin{array}{l}0.060 \\
(0.071)\end{array}$ & $\begin{array}{l}0.088 \\
(0.071)\end{array}$ & $\begin{array}{l}-0.145^{*} \\
(0.061)\end{array}$ & $\begin{array}{l}-0.064 \\
(0.061)\end{array}$ \\
\hline Constant & $\begin{array}{l}-50.651 \\
(34.898)\end{array}$ & $\begin{array}{l}-53.071 \\
(34.912)\end{array}$ & $\begin{array}{l}-106.398 * \\
(49.570)\end{array}$ & $\begin{array}{l}-108.520^{*} \\
(49.577)\end{array}$ & $\begin{array}{l}-51.979^{+} \\
(30.738)\end{array}$ & $\begin{array}{l}-54.134^{+} \\
(30.729)\end{array}$ \\
\hline BRD $(\operatorname{In}) *$ localized $_{t-1}$ & $\begin{array}{l}-0.001 \\
(0.079)\end{array}$ & $\begin{array}{l}-0.001 \\
(0.079)\end{array}$ & $\begin{array}{l}-0.122 \\
(0.092)\end{array}$ & $\begin{array}{l}-0.122 \\
(0.092)\end{array}$ & $\begin{array}{l}0.084 \\
(0.069)\end{array}$ & $\begin{array}{l}0.084 \\
(0.069)\end{array}$ \\
\hline Greenfield $\mathrm{FDI}_{\mathrm{i}, t-1}(\mathrm{In})$ & $\begin{array}{l}0.120^{* *} \\
(0.037)\end{array}$ & $\begin{array}{l}0.120^{* *} \\
(0.037)\end{array}$ & $\begin{array}{l}0.037 \\
(0.033)\end{array}$ & $\begin{array}{l}0.037 \\
(0.033)\end{array}$ & $\begin{array}{l}0.137^{\star * *} \\
(0.036)\end{array}$ & $\begin{array}{l}0.137^{* * *} \\
(0.036)\end{array}$ \\
\hline $\mathrm{GDP}_{t-1}$ & $\begin{array}{l}-0.100 \\
(0.460)\end{array}$ & $\begin{array}{l}-0.100 \\
(0.460)\end{array}$ & $\begin{array}{l}-0.933 \\
(0.655)\end{array}$ & $\begin{array}{l}-0.933 \\
(0.655)\end{array}$ & $\begin{array}{l}0.293 \\
(0.442)\end{array}$ & $\begin{array}{l}0.293 \\
(0.442)\end{array}$ \\
\hline Population $_{t-1}(\ln )$ & $\begin{array}{l}3.955 \\
(2.417)\end{array}$ & $\begin{array}{l}3.955 \\
(2.417)\end{array}$ & $\begin{array}{l}7.898^{\star} \\
(3.316)\end{array}$ & $\begin{array}{l}7.898^{*} \\
(3.316)\end{array}$ & $\begin{array}{l}3.761^{+} \\
(2.155)\end{array}$ & $\begin{array}{l}3.761^{+} \\
(2.155)\end{array}$ \\
\hline $\begin{array}{l}\text { WGI regulatory } \\
\text { quality }_{t-1}\end{array}$ & $\begin{array}{l}0.275 \\
(0.706)\end{array}$ & $\begin{array}{l}0.275 \\
(0.706)\end{array}$ & $\begin{array}{l}1.091 \\
(1.017)\end{array}$ & $\begin{array}{l}1.091 \\
(1.017)\end{array}$ & $\begin{array}{l}-0.454 \\
(0.620)\end{array}$ & $\begin{array}{l}-0.454 \\
(0.620)\end{array}$ \\
\hline Polity index ${ }_{t-1}$ & $\begin{array}{l}-0.037 \\
(0.044)\end{array}$ & $\begin{array}{l}-0.037 \\
(0.044)\end{array}$ & $\begin{array}{l}0.008 \\
(0.047)\end{array}$ & $\begin{array}{l}0.008 \\
(0.047)\end{array}$ & $\begin{array}{l}-0.050 \\
(0.036)\end{array}$ & $\begin{array}{l}-0.050 \\
(0.036)\end{array}$ \\
\hline Exchange rate $_{t-1}(\mathrm{In})$ & $\begin{array}{l}-0.400 \\
(0.295)\end{array}$ & $\begin{array}{l}-0.400 \\
(0.295)\end{array}$ & $\begin{array}{l}0.050 \\
(0.459)\end{array}$ & $\begin{array}{l}0.050 \\
(0.459)\end{array}$ & $\begin{array}{l}-0.319 \\
(0.295)\end{array}$ & $\begin{array}{l}-0.319 \\
(0.295)\end{array}$ \\
\hline $\begin{array}{l}\text { Exchange rate } \\
\text { volatility }_{t-1}\end{array}$ & $\begin{array}{l}-13.326^{*} \\
(5.404)\end{array}$ & $\begin{array}{l}-13.326^{*} \\
(5.404)\end{array}$ & $\begin{array}{l}-6.163 \\
(7.057)\end{array}$ & $\begin{array}{l}-6.163 \\
(7.057)\end{array}$ & $\begin{array}{l}-4.232 \\
(3.556)\end{array}$ & $\begin{array}{l}-4.232 \\
(3.556)\end{array}$ \\
\hline $\begin{array}{l}\text { Control of } \\
\text { corruption }_{t-1}\end{array}$ & $\begin{array}{l}1.000^{+} \\
(0.568)\end{array}$ & $\begin{array}{l}1.000^{+} \\
(0.568)\end{array}$ & $\begin{array}{l}0.361 \\
(0.778)\end{array}$ & $\begin{array}{l}0.361 \\
(0.778)\end{array}$ & $\begin{array}{l}1.117^{*} \\
(0.542)\end{array}$ & $\begin{array}{l}1.117^{\star} \\
(0.542)\end{array}$ \\
\hline Inflation $_{t-1}$ & $\begin{array}{l}0.001 \\
(0.008)\end{array}$ & $\begin{array}{l}0.001 \\
(0.008)\end{array}$ & $\begin{array}{l}0.011 \\
(0.010)\end{array}$ & $\begin{array}{l}0.011 \\
(0.010)\end{array}$ & $\begin{array}{l}-0.002 \\
(0.007)\end{array}$ & $\begin{array}{l}-0.002 \\
(0.007)\end{array}$ \\
\hline Observations & 1234 & 1234 & 1234 & 1234 & 1234 & 1234 \\
\hline Number of countries & 90 & 90 & 90 & 90 & 90 & 90 \\
\hline Country FE & Yes & Yes & Yes & Yes & Yes & Yes \\
\hline Year FE & Yes & Yes & Yes & Yes & Yes & Yes \\
\hline
\end{tabular}

Note: Political conflict proxied by battle-related deaths. Robust standard errors in parentheses.

${ }^{* * *} p<0.001,{ }^{* *} p<0.01,{ }^{*} p<0.05,{ }^{+} p<0.10$. 